\title{
Can subthreshold summation be observed with the Ehrenstein illusion?
}

\author{
Véronique Salvano-Pardieu, Brian Wink $\uparrow$, Alain Taliercio, Ken Manktelow§, \\ Thomas Meigen\# \\ Département de Psychologie, Laboratoire UPRES 2114, Université de Tours, 3 rue des Tanneurs, \\ BP 4103, F 37041 Tours Cedex 1, France; e-mail: veronique.pardieu@wanadoo.fr; Psychology \\ Group, Southampton Solent University, East Park Terrace, Southampton SO14 0YN, UK; \\ e-mail: Brian.Wink@solent.ac.uk; § Psychology Division, University of Wolverhampton, Wulfruna Street, \\ Wolverhampton WV1 1SB, UK; \# Universitäts-Augenklinik, Josef-Schneider-Strasse 11, D 97080 Würzburg, \\ Germany
}

Received 3 December 2003, in revised form 27 June 2005; published online 9 June 2006

\begin{abstract}
Subthreshold summation between physical target lines and illusory contours induced by edges such as those produced in the Kanizsa illusion has been reported in previous studies. Here, we investigated the ability of line-induced illusory contours, using Ehrenstein figures, to produce similar subthreshold summation. In the first experiment, three stimulus conditions were presented. The target line was superimposed on the illusory contour of a four-arm Ehrenstein figure, or the target was presented between two dots (which replaced the arms of the Ehrenstein figure), or the target was presented on an otherwise blank screen (control). Detection of the target line was significantly worse when presented on the illusory contour (on the Ehrenstein figure) than when presented between two dots. This result was consistent for both curved and straight target lines, as well as for a $100 \mathrm{~ms}$ presentation duration and unlimited presentation duration. Performance was worst in the control condition. The results for the three stimulus conditions were replicated in a second experiment in which an eight-arm Ehrenstein figure was used to produce a stronger and less ambiguous illusory contour. In the third experiment, the target was either superimposed on the illusory contour, or was located across the central gap (illusory surface) of the Ehrenstein figure, collinear with two arms of the figure. As in the first two experiments, the target was either presented on the Ehrenstein figure, or between dots, or on a blank screen. Detection was better in the dot condition than in the Ehrenstein condition, regardless of whether the target was presented on the illusory contour or collinear with the arms of the Ehrenstein figure. These three experiments demonstrate the ability of reduced spatial uncertainty to facilitate the detection of a target line, but do not provide any evidence for subthreshold summation between a physical target line and the illusory contours produced by an Ehrenstein figure. The incongruence of these results with previous findings on Kanizsa figures is discussed.
\end{abstract}

\section{Introduction}

\subsection{The properties of illusory figures}

The perception of form is commonly based on contours defined by steps in the luminance profile, but it can also occur in the absence of a physical stimulus in so-called illusory figures, which give rise to illusory contours. Prominent demonstrations of illusory contours are the figures of Ehrenstein (1941/1987) and of Kanizsa (1955/1987). Previous studies have demonstrated that two components of the Ehrenstein illusion (see figure 1) can be distinguished: illusory brightness and clarity of contour. Illusory brightness has been investigated by several researchers (Spillmann 1975; Kennedy 1976; Dresp et al 1996; Salvano-Pardieu 2000), and is defined as a perceived difference between the brightness of surfaces that occurs in the absence of luminance differences. For example, the central gap in figure 1 appears to differ in brightness from the background. The brightness illusion is observed with collinear opposition of the inducers. For example, in the Ehrenstein illusion, inducers are collinear two-by-two across the central gap and allow neon spreading in the central gap (Redies et al 1984). In contrast, the shape of the contour is not collinear with the inducers. Clarity of contour is affected by the 


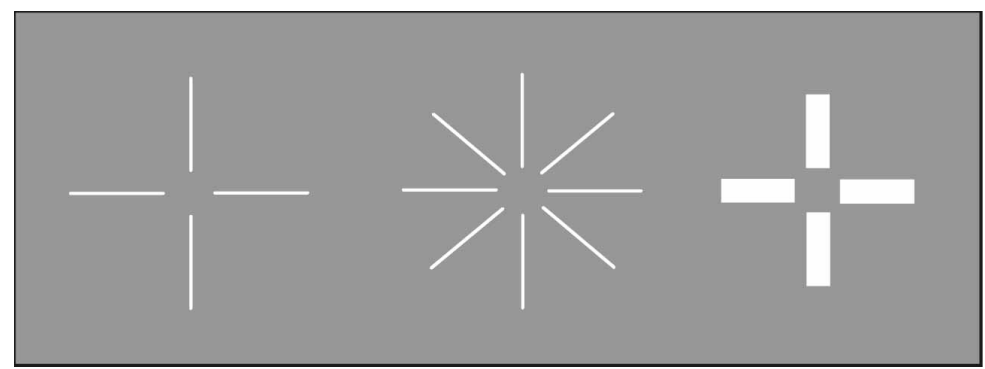

Figure 1. Ehrenstein figure. An illusory figure induced by end-lines showing an illusory contour and an illusory surface. The form of the illusory contour in the Ehrenstein figure changes as a function of the number and shape of the inducers.

shape of the figure. An illusory contour is a contour that is perceived in a figure in the absence of local stimulus information. The shape of the illusory contour can be modified by changing the spatial position of the inducing elements. For example, in the Ehrenstein figure, the shape of the illusory contour can be modified by changing the spatial position of the inducing arms (Spillmann 1975; Hamada and Nakahashi 1995). Furthermore, in an Ehrenstein figure generated by only four arms (figure 1) the shape of the illusory contour is ambiguous, in that some observers report a diamond illusory shape while others report a circle or a light blob with no specific shape (Coren et al 1987). As shown by Hamada and Nakahashi (1995), this ambiguity disappears when more arms are added to the Ehrenstein figure and when the space between two inducers is reduced.

The effects of the luminance and contrast polarity of the inducers have been investigated in a number of studies of illusory contours. It has been shown that an illusory configuration can be generated by inducers of opposite contrast polarity (Prazdny 1983; Grossberg and Mingolla 1985a; Shapley and Gordon 1987), although the illusory brightness is weakened if not abolished (Spillmann et al 1984; Kellman and Loukides 1987; Dresp et al 1996). These studies have also shown that local contrast between inducers and the background is involved in the formation of illusory brightness, but not in the formation of illusory contours. Thus, illusory contours may be mediated by a mechanism that is able to integrate opposite contrast polarities across space. The earliest research on the effects of different contrast polarities on illusory-contour formation was mainly based on qualitative observations (Prazdny 1983). More recent studies have used psychophysical methods to provide evidence that illusory contours are visible in isoluminance (Ejima and Takahashi 1988), and that inducers of different contrast polarity can be integrated in the formation of a Kanizsa-square illusory contour (Dresp and Grossberg 1997, 1999).

Most neurophysiological models try to explain illusory-contour formation in terms of the properties of the receptive fields of cortical cells (eg Peterhans and von der Heydt 1989; Grossberg 1994; Gove et al 1995; Heider et al 2002). These models mainly consist of neural networks with weighted connections between units. Thus, an illusory line or edge could be generated by the activities of detectors or groups of neurons that process the orientation information of the stimulus. Dresp and Grossberg (1997, 1999) have shown that illusory-contour integration may depend on long-range interactions across visual space.

\subsection{Subthreshold summation}

Kulikowski and King-Smith (1973) showed that the detection threshold of a target line presented on a subliminal stimulus line was reduced relative to the detection threshold of a real line presented alone. This was explained in terms of summation 


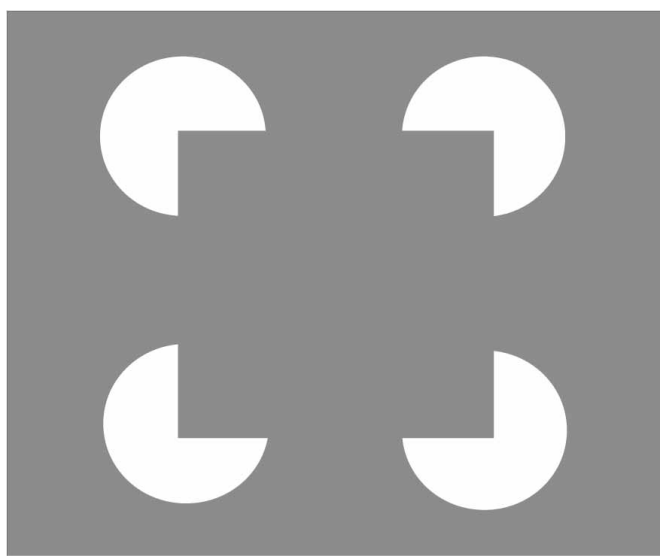

Figure 2. Kanizsa square. An illusory figure induced by edges, showing an illusory contour and an illusory surface.

between the two targets, and is referred to as subthreshold summation. Using a Kanizsa square (figure 2), Dresp and Bonnet (1995) reported a similar reduction in the detection threshold of a target line when it was superimposed on an illusory contour rather than on a subliminal line, in a study with two participants. When the target line was presented on the illusory contour, between two inducers (pacmen in the Kanizsa square), the detection threshold of the target line was found to be lower than the detection threshold of a line presented alone or between two spatial cues that did not generate an illusory contour (such as two arrowheads). More recently, Poom (2001) confirmed these results with configurations generating either illusory brightness or illusory contours. These authors reported that spatial cues that did not generate illusory contours did not facilitate detection as effectively as the illusory contours. Subthreshold summation with Kanizsa-square illusory contours has also been reported by Dresp and Grossberg (1997, 1999), in studies with inducers of different contrast polarity (black and white pacmen) and different colours (green and red pacmen).

The existence of subthreshold summation suggests that the illusory contour shares some of the characteristics of a real contour (see Lesher 1995; Spillmann and Dresp 1995, for reviews). Subthreshold summation between illusory contours and real contours is congruent with the hypotheses of the neurophysiological models of Grossberg and Mingolla (1985a, 1985b), Grossberg (1994), and Gove et al (1995). Evidence also exists for the involvement of higher cortical areas in the perception of illusory contours. For example, Huxlin et al (2000) have shown that lesions to the inferotemporal cortex of monkeys impair their ability to see illusory contours.

\subsection{Subthreshold summation and the Ehrenstein illusion}

As we have seen, the subthreshold summation paradigm has previously been used to investigate the Kanizsa square (Dresp and Bonnet 1995; Dresp and Grossberg 1997, 1999), in which the illusory contours are generated by edges, and the inducers are collinear with the illusory contour. If we assume that similar underlying mechanisms mediate the illusory contours observed in both Kanizsa and Ehrenstein figures, then it seems reasonable to assume that subthreshold summation will occur with both edge induced and line-end induced contours. This is congruent with the characteristics of the bipole filter described in physiological models (eg Grossberg and Mingolla 1985a, 1985b; Grossberg 1994; Gove et al 1995) and with previous explanations of subthreshold summation between physical targets and illusory contours (Dresp and Bonnet 1995). According to this explanation, detection should be facilitated by the illusory contour when a target joins two adjacent arms of the figure. Such a model does not require the inducers to be collinear for the putative bipole filters to be activated. In many previous studies only half of a Kanizsa figure was investigated. In this study complete 
Ehrenstein figures are used. When the target joins two adjacent arms of the Ehrenstein figure, in addition to facilitation of detection as a result of subthreshold summation with the illusory contour, we might expect detection to be improved by a reduction in spatial uncertainty. In both the Kanizsa figure and the Ehrenstein figure the inducers provide an accurate cue to the location of the target. This, in itself, should make detection of the target easier than when the target is presented alone. This, therefore, needs to be controlled for. In this study this is done by replacing the inducing arms of the Ehrenstein figure with small dots. The dots clearly indicate the location of the target, but generate no illusory contour. If detection is facilitated by the reduced spatial uncertainty, then performance will be better with dots than with no cue (blank screen). If subthreshold summation occurs between the illusory contour and the target, then the Ehrenstein figure will facilitate detection more than the dots alone.

\section{Experiment 1}

In the first experiment participants were required to detect a target line. The target was either superimposed on the illusory contour generated by a four-arm Ehrenstein figure, or the target was located between dots which replaced the arms of the Ehrenstein figure, or the target was presented alone on an otherwise blank screen. The dots indicate the possible location of the target without generating an illusory contour.

The shape of the target line was either straight or curved, in order to superimpose the ambiguous diamond or circular illusory contours, respectively, generated by a fourarm Ehrenstein figure. In addition, two different durations of target presentation were compared: $100 \mathrm{~ms}$ and unlimited. The duration of $100 \mathrm{~ms}$ was chosen to prevent participants from fixating all possible target locations. A square or circular mask was used to cancel any afterimage in the 100-ms-duration condition. In the unlimited-duration condition, the target was presented until the observers responded. No interaction between stimulus condition and presentation duration was expected.

\subsection{Method}

2.1.1 Participants. Eight participants (three male and five female, aged between 22 and 35 years) took part in this experiment. All but one were naive as to the purpose of the present study, and all but three had already taken part or been trained in psychophysical tasks. All had normal or corrected-to-normal acuity.

2.1.2 Stimuli and experimental design. The stimuli were viewed binocularly on a monochrome computer screen with a resolution of $640 \times 480$ pixels $(67 \mathrm{~Hz}$, non-interlaced). They were generated with a Power Macintosh (8600/250). As mentioned earlier, there were three stimulus conditions (Ehrenstein, dot, and control). In the first condition, an Ehrenstein figure with four orthogonal lines of equal length in a collinear two-by-two configuration was presented. This figure generated clear illusory contours. In the second condition, four dots were presented instead of the lines; these produced no illusory contours. In the final, control, condition, the target was presented alone.

In each condition participants were required to detect two kinds of target lines: straight target lines or curved target lines, and these were presented for either $100 \mathrm{~ms}$ or unlimited time (self-paced, until the participant's response). Orthogonal crossing of the three factors (stimulus condition, target shape, and presentation duration) gave twelve conditions (see figure 3). Each of the twelve conditions was completed separately in a balanced order, which was varied between participants. The stimuli were presented on a grey background with a luminance of $40.15 \mathrm{~cd} \mathrm{~m}^{-2}$. The width of the lines in the Ehrenstein condition was $1.5 \mathrm{~min}$ of arc and their length was $2 \mathrm{deg}$ of visual angle. In the dot condition, a dot with a diameter of $1.5 \mathrm{~min}$ of arc replaced each line. The luminance of the inducing lines and the dots was $80 \mathrm{~cd} \mathrm{~m}^{-2}$. The central gap size of the figures in both the Ehrenstein and the dot conditions was $2 \mathrm{deg}$. 


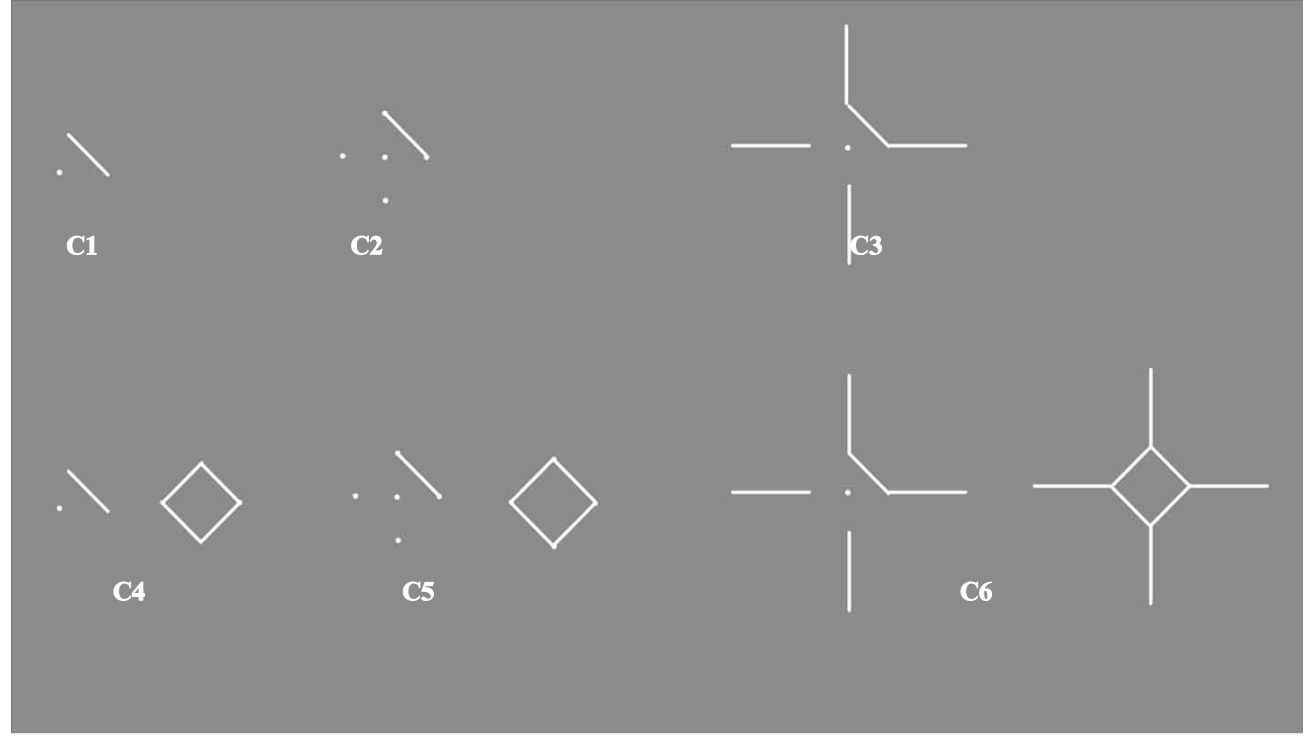

(a)

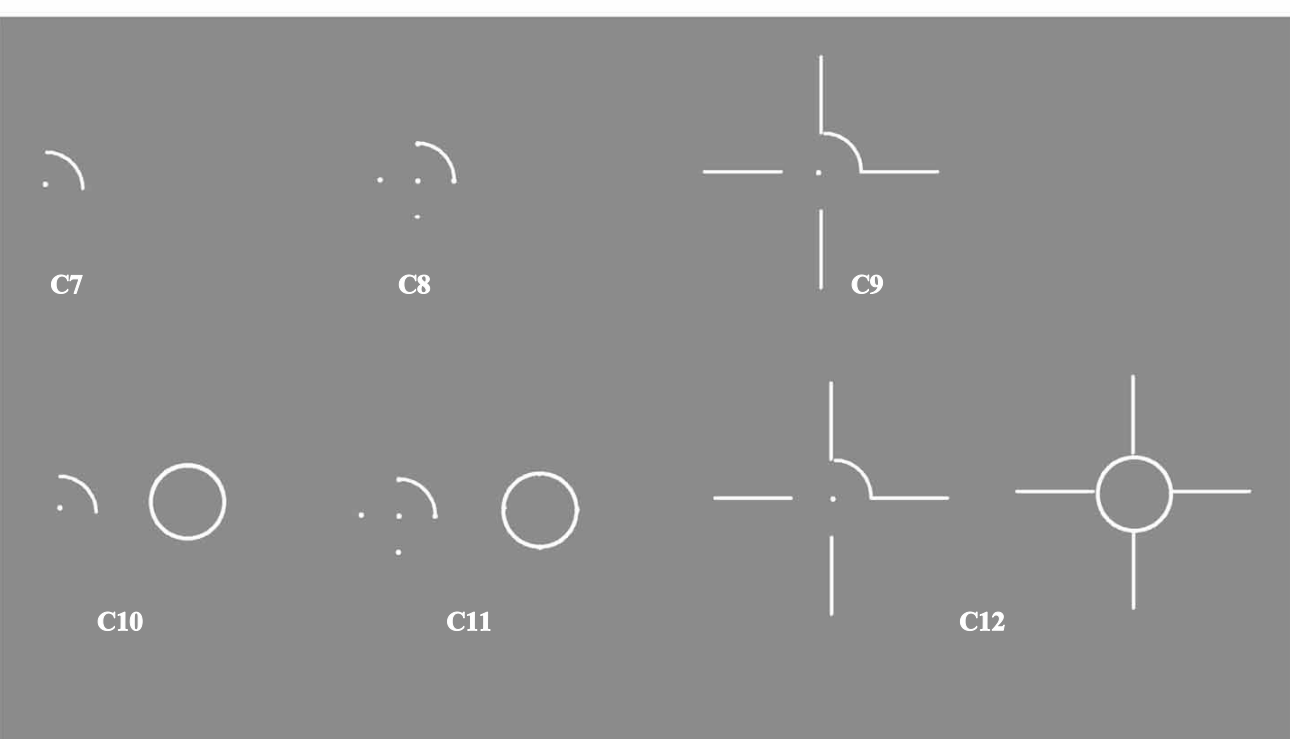

(b)

Figure 3. The twelve stimulus conditions used in experiment 1. Subjects had to detect a target line that was brighter than the background. The target line was either presented alone in the control condition (C1), or joining two dots in a four-dot condition (C2), or superimposed on the illusory contour and joining the terminations of two inducers in the Ehrenstein condition (C3). These three conditions ( 1 1, C2, and C3) were presented for an unlimited time or for a short presentation time (100 ms) followed by a diamond mask (C4, C5, and C6). These six conditions with a straight target line are shown in (a). The other six conditions with a curved target line are shown in (b). Except for the shape of the target line, all other characteristics of the stimuli were unchanged. The presentation time was either unlimited (C7, C8, C9), or short (100 ms), followed by a circular mask $(\mathrm{C} 10, \mathrm{C} 11, \mathrm{C} 12)$.

The target line had a width of $1.5 \mathrm{~min}$ of arc and a length of $1.4 \mathrm{deg}$ and connected the terminations of two lines or two dots. In the control condition, only the target line was presented to the subjects. On each trial the target line appeared at the top-right, the top-left, the bottom-right, or the bottom-left of a central fixation point 
of $1.5 \mathrm{~min}$ of arc, as shown in figure 4 . When the presentation duration of the target line was unlimited, the inducing figure or dots and the target remained on the screen until the participants gave their response. In the 100-ms conditions the target line was replaced by a mask which superimposed all 4 possible target positions after $100 \mathrm{~ms}$. The mask formed a diamond shape when the target was a straight line, and was a circle when the target was curved (see figure 3). The mask remained on the screen until the participant responded. A presentation duration of $100 \mathrm{~ms}$ was chosen to allow participants to detect the target, but prevent them from moving their eyes to search for it.
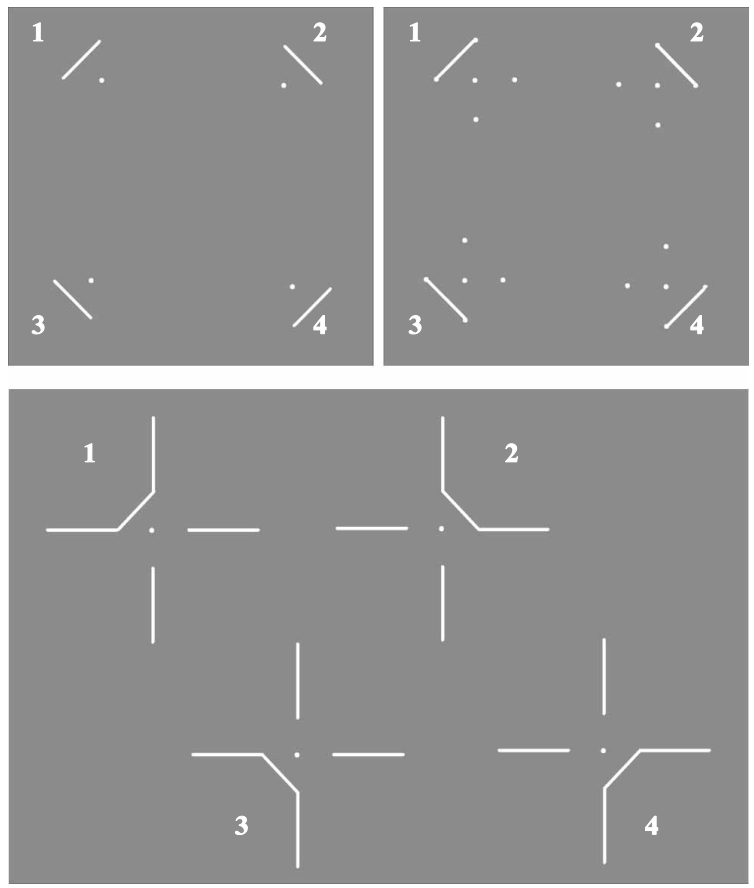

Figure 4. On each trial the target line appeared at the top-right, top-left, bottom-right, or bottom-left of a central fixation point of $1.5 \mathrm{~min}$ of arc diameter. Only the straight target lines are shown.

Before each trial, a fixation point with a luminance of $60 \mathrm{~cd} \mathrm{~m}^{-2}$ was presented in the centre of the screen together with a $500 \mathrm{~Hz}$ tone for $100 \mathrm{~ms}$. Participants viewed the screen from $70 \mathrm{~cm}$, with the head stabilised by a chin-rest. The only source of light in the room, in addition to that of the screen, was a $60 \mathrm{~W}$ bulb placed on the floor to provide diffuse ambient illumination at a photopic level, keeping the illumination level of the room constant throughout the experiment.

2.1.3 Procedure. An adaptive best-PEST (Pentland 1980; Lieberman and Pentland 1982) four-alternative forced-choice (4AFC) procedure was used for threshold determination. Participants were asked to indicate whether the target appeared at the top-right, top-left, bottom-right, or bottom-left of the stimulus figure by pressing the $9,7,3$, and 1 keys of the numeric computer keypad, respectively. The initial luminance of the target line was $60 \mathrm{~cd} \mathrm{~m}^{-2}$ and this was either decreased following a correct answer or increased following an incorrect answer according to the best-PEST procedure. The threshold was calculated after 45 presentations. Each condition was presented four times (session 1 to 4). The entire experiment lasted approximately $2 \mathrm{~h}$ for each participant and was divided into two 1-h sessions. 


\subsection{Results}

The results were analysed with a four-way repeated-measures analysis of variance (ANOVA): stimulus condition (Ehrenstein, dot, or control), target shape (straight or curved), presentation duration (mask or unlimited), and session (1 to 4).

2.2.1 Main effect of stimulus condition. As shown in figure 5 the threshold for target detection was lower in the dot condition $\left(41.62 \mathrm{~cd} \mathrm{~m}^{-2}\right)$ than in the Ehrenstein condition $\left(41.76 \mathrm{~cd} \mathrm{~m}^{-2}\right)$, and highest in the control condition $\left(42.17 \mathrm{~cd} \mathrm{~m}^{-2}\right)$. The ANOVA shows a significant main effect of stimulus condition $\left(F_{2,22}=15.23, p<0.01\right)$. Furthermore, planned comparisons show that the detection threshold in the dot condition was significantly lower than in the Ehrenstein condition $\left(F_{1,11}=22.88, p<0.01\right)$, whilst the threshold in the control condition was significantly higher than in the Ehrenstein condition.

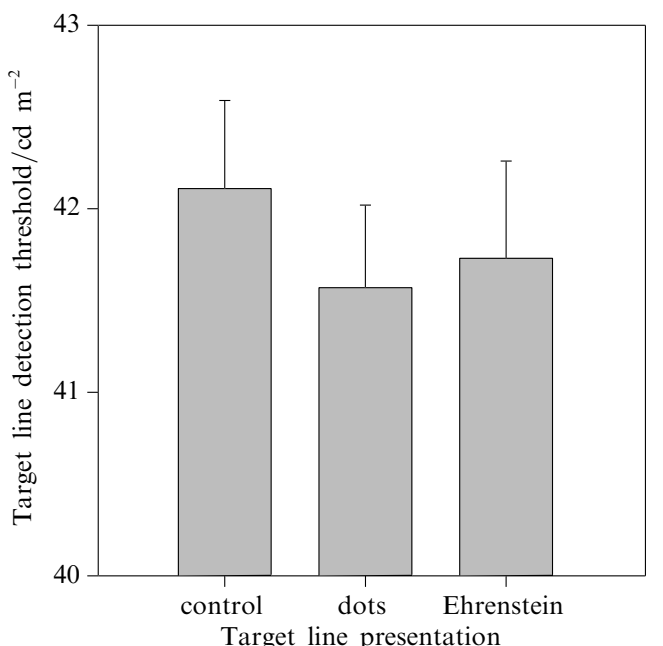

Figure 5. Mean detection thresholds and standard errors for the three experimental conditions (control, dots, Ehrenstein). Results are averaged across eight participants.

2.2.2 Main effect of target shape. The overall detection threshold for a curved target line $\left(41.76 \mathrm{~cd} \mathrm{~m}^{-2}\right)$ was not significantly different from the detection threshold for the straight target line $\left(41.94 \mathrm{~cd} \mathrm{~m}^{-2}\right)\left(F_{1,11}=1.66, \mathrm{~ns}\right)$.

2.2.3 Stimulus-condition/target-shape interaction. An interaction was found between stimulus condition and target shape $\left(F_{2,22}=4.25, p<0.05\right)$. Whilst the curved target always had a lower detection threshold than the straight target, as shown in figure 6 , this difference was only significant in the Ehrenstein condition. With the curved target there was no significant difference between the dot condition $\left(41.55 \mathrm{~cd} \mathrm{~m}^{-2}\right)$ and the Ehrenstein condition $\left(41.58 \mathrm{~cd} \mathrm{~m}^{-2}\right)$. In contrast, with the straight target the difference between the dot $\left(41.59 \mathrm{~cd} \mathrm{~m}^{-2}\right)$ and Ehrenstein $\left(41.89 \mathrm{~cd} \mathrm{~m}^{-2}\right)$ conditions was significant $\left(F_{2,14}=8.25, p<0.01\right)$, with performance enhanced in the dot condition.

2.2.4 Main effect of presentation duration. The performance of participants was better when the presentation duration was unlimited $\left(41.48 \mathrm{~cd} \mathrm{~m}^{-2}\right)$, than when it was $100 \mathrm{~ms}$ $\left(42.22 \mathrm{~cd} \mathrm{~m}^{-2}\right)\left(F_{1,11}=184.32, p<0.01\right)$. As expected, the same pattern of results was found for all stimulus conditions (Ehrenstein, dot, and control), regardless of presentation duration. As shown in figure 7, higher thresholds were found at $100 \mathrm{~ms}$ than for unlimited presentation in all stimulus conditions. No significant interaction was found between presentation duration and stimulus condition. In addition, no interaction was found between target shape and presentation duration. Finally, no main effect or interactions were obtained for session. 


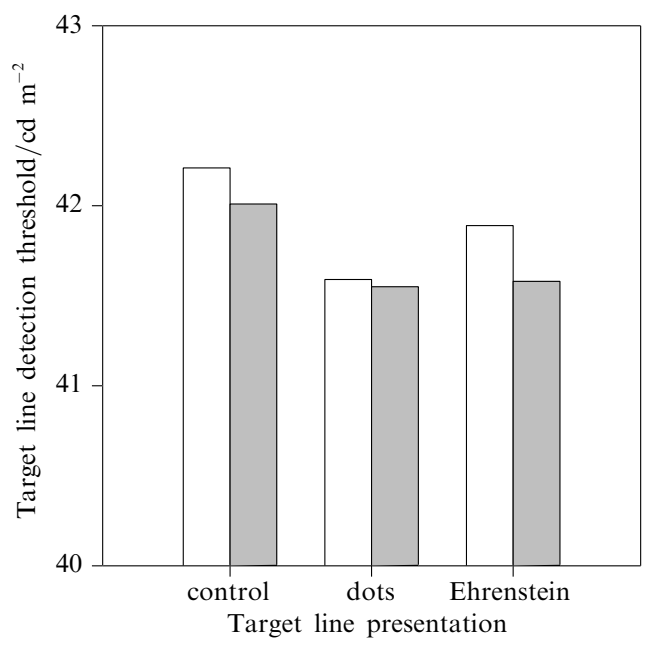

$\square$ straight target line

Figure 6. Mean detection thresholds for target line shape (straight and curved) and condition (control, dots, Ehrenstein). Results are averaged across eight participants.

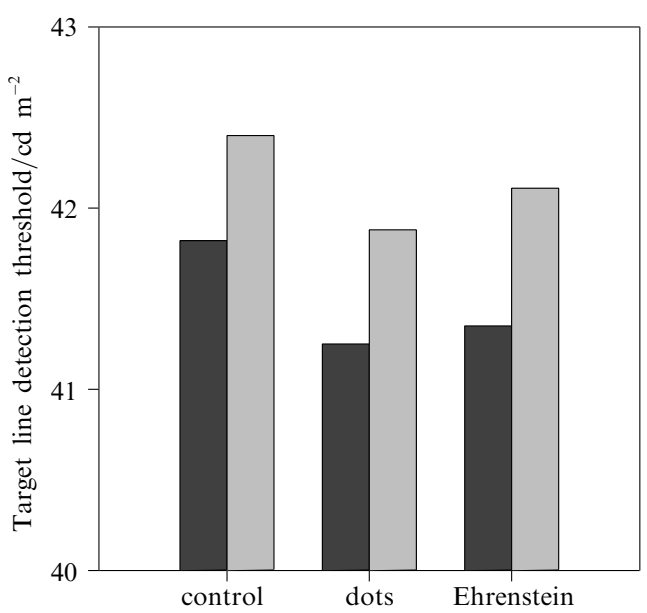

unlimited presentation time short presentation time

Figure 7. Mean detection thresholds for presentation duration (unlimited and short) and condition (control, dots, Ehrenstein). Results are averaged across eight participants.

\subsection{Discussion}

These results show that performance in the Ehrenstein condition was significantly worse than in the dot condition. This is in contrast to the prediction that subthreshold summation between the target and the illusory contour (present in the Ehrenstein figure), would lead to a facilitation of detection in the Ehrenstein condition. There was no improvement in detection with either straight or curved targets designed to superimpose the illusory contour of the Ehrenstein figure. In fact, performance was enhanced when the arms of the Ehrenstein figure were replaced by dots, which do not generate an illusory contour.

One reason for the failure to find evidence for subthreshold summation in this experiment may be the choice of figure. In a four-arm Ehrenstein figure, the illusory contour is relatively weak, and as discussed earlier it is ambiguous and may appear as either a circle or a diamond (Deters-Brüggemann and Meinhardt 1994). A straight target line joining two inducers would perfectly superimpose a diamond-shaped illusory contour, but would only partially overlap with a circular contour. Likewise, a curved target line would only partially overlap with a straight illusory contour. If it is assumed that the summation is mediated by the overlap of the receptive fields of neurons involved in the detection of the physical target line and the receptive fields involved in the generation of the illusory contour, then, if the shape of the contour and that of the 
target are different, any summation is likely to be weaker. It is therefore possible that the clarity and stability of the contour in experiment 1 was insufficient to elicit subthreshold summation. To investigate this possibility a second experiment was conducted, with an eight-arm Ehrenstein figure. This figure generates a stronger, unambiguous, diamond-shaped illusory contour (see figure 8) and will allow a direct comparison with the stimuli used in experiment 1 . The target in this second experiment is the same as that used in the straight-line condition in the first experiment.

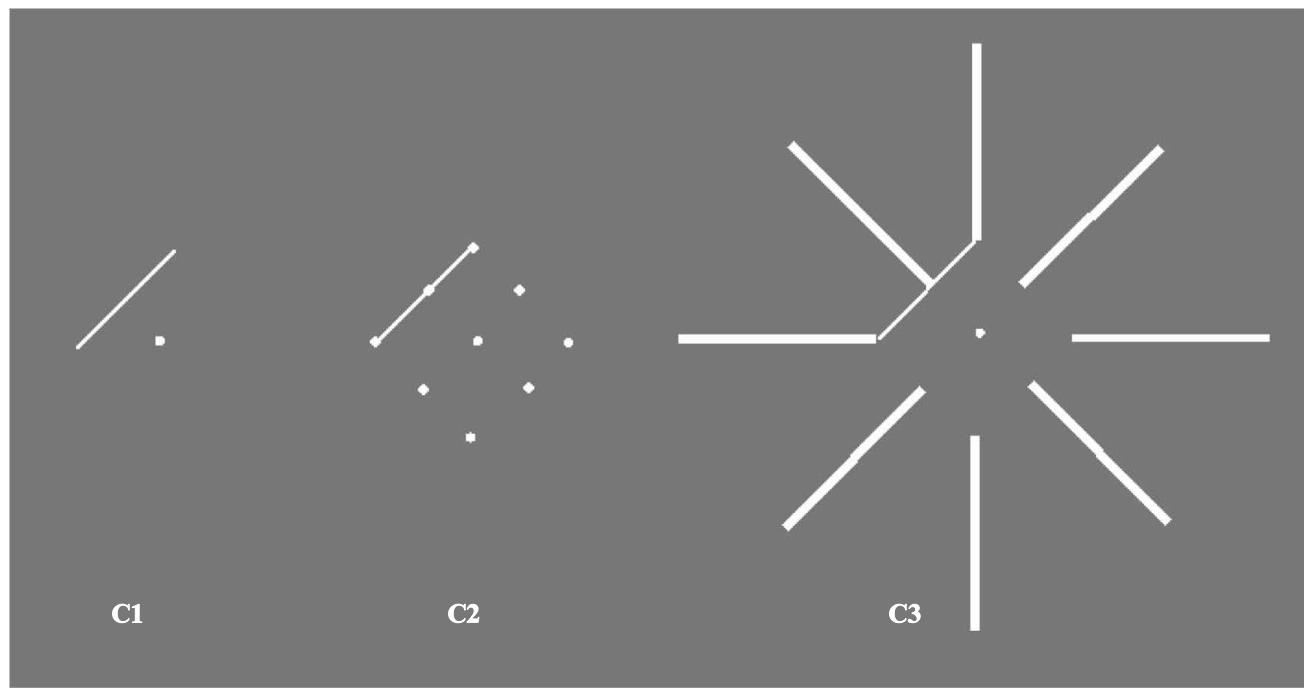

Figure 8. The three stimulus conditions used in experiment 2. As in the previous experiment, subjects had to detect a target line that was brighter than the background either presented alone in the control condition $(\mathrm{C} 1)$, or joining two dots in an eight-dot condition (C2), or superimposed on the illusory contour and joining the terminations of two inducers in the Ehrenstein condition (C3). In this experiment the eight-arm Ehrenstein figure generated a clear diamondshaped illusory contour.

\section{Experiment 2}

\subsection{Method}

3.1.1 Participants. Seven participants (one male and six female, aged between 22 and 35 years) took part in this experiment. All were naive as to the purpose of the present study, and all but three had previously taken part in psychophysical tasks. All had normal or corrected-to-normal acuity.

3.1.2 Stimuli and experimental design. The stimuli were viewed binocularly on a monochrome computer screen and were generated with a Cambridge Research Systems VSG 2/3 graphics card. All stimulus characteristics (length, width, size, luminance, etc) and the experimental design (distance from the screen, illumination level of the room, fixation point, etc) were similar to those used in experiment 1 . However, the Ehrenstein figure was generated by eight arms instead of four, and consequently generated a clear diamond-shaped illusory contour. In the dot condition, there were eight dots in place of the arms of the Ehrenstein figure, which produced no illusory contour. As in experiment 1 , in the control condition the target was alone on a blank screen. Since the illusory contours were unambiguously straight in this experiment, the target lines were always straight. As the pattern of results was the same for both presentation durations in experiment 1, only the unlimited presentation duration was investigated in this experiment. The observers viewed each of the three stimulus conditions in a random order. The stimuli can be seen in figure 8 . 
3.1.3 Procedure. A 4AFC classical staircase procedure was used for threshold determination. While this threshold calculation method varies from the best-PEST estimation, no significant difference has been found when comparing classical staircase and bestPEST estimations in computer simulation of measures of subjective equality (Meese 1995). We therefore expect comparable results, regardless of the threshold calculation method employed. Participants were asked to detect a target line, either to the top-right, top-left, bottom-right, or bottom-left of a fixation point. They indicated their response on a four-key response box (Cambridge Research Systems CT3), on which the keys are arranged in a two-by-two fashion, which coincided with the target positions (top-right, top-left, bottom-right, and bottom-left). The initial luminance of the target line was $60 \mathrm{~cd} \mathrm{~m}^{-2}$, and this was either decreased following a correct answer or increased following an incorrect answer according to the staircase procedure. The steps initially changed by $5 \mathrm{~cd} \mathrm{~m}^{-2}$ the luminance of the target until it reached $45 \mathrm{~cd} \mathrm{~m}^{-2}$ then they changed it by $0.25 \mathrm{~cd} \mathrm{~m}^{-2}$ until it reached $42 \mathrm{~cd} \mathrm{~m}^{-2}$, after which they changed it by $0.05 \mathrm{~cd} \mathrm{~m}^{-2}$ until the staircase terminated. This adjustment in the step size reduces the length of the experiment for the participant whilst providing accurate measurements close to threshold. The threshold was calculated following three errors within 10 trials. The staircase was programmed to stop after 70 trials if a threshold had not been reached, but this did not occur for any of the participants. As in experiment 1 , each condition was presented four times. The entire experiment lasted approximately $20 \mathrm{~min}$.

\subsection{Results}

The results were analysed with a two-way repeated-measures ANOVA: stimulus condition (Ehrenstein, dot, or control) and session (1 to 4).

3.2.1 Main effect of stimulus condition. The threshold for target detection was lower in the dot condition $\left(41.02 \mathrm{~cd} \mathrm{~m}^{-2}\right)$, than in the Ehrenstein condition $\left(41.34 \mathrm{~cd} \mathrm{~m}^{-2}\right)$, and highest in the control condition $\left(41.48 \mathrm{~cd} \mathrm{~m}^{-2}\right)$. This can be seen in figure 9. The ANOVA shows a significant main effect of stimulus condition $\left(F_{2,12}=8.19, p<0.01\right)$. Planned comparisons show that the detection threshold in the dot condition was significantly lower than in the Ehrenstein condition $\left(F_{1,6}=8.19, p<0.05\right)$. However, the difference between the Ehrenstein and control conditions was not significant $\left(F_{1,6}=2.56, p>0.05\right)$. No main effect or interactions were obtained for session.

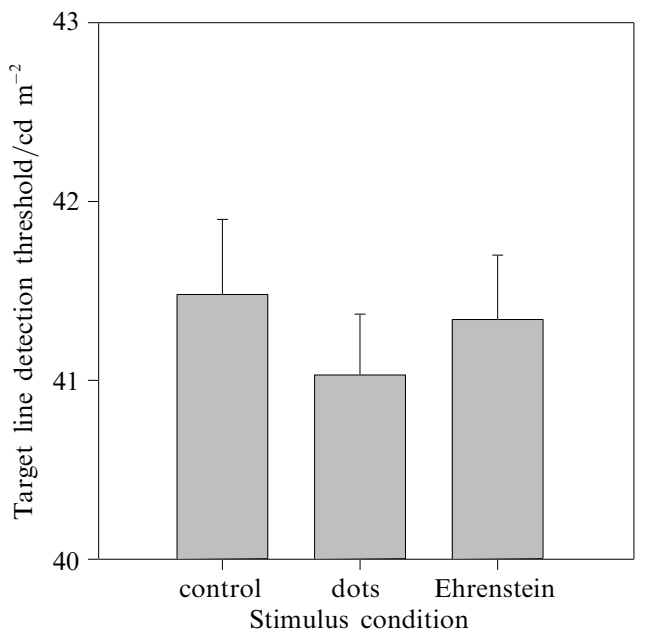

Figure 9. Mean detection thresholds and standard errors for the three experimental conditions (control, dots, Ehrenstein). Results are averaged across seven participants. 


\subsection{Discussion}

Despite the use of an eight-arm Ehrenstein figure in experiment 2, the results show no evidence of subthreshold summation. As in the first experiment, the results suggest that target detection may be facilitated by cuing the location of the target, rather than the presence of the illusory contour. In both experiments, the arms of the Ehrenstein figure and the dots in the dot condition clearly indicate to observers where they should look to detect the target. In both experiments, detection was found to be better in the dot condition, where the target was located between two dots, than in the Ehrenstein condition, where the target was superimposed on the illusory contour. Hence, detection was actually better in the absence of the illusory contour. In both experiments the worst performance was observed in the control condition, where the target was presented on a blank screen and there were no cues to the target location. In the control condition, spatial uncertainty is therefore much greater, making detection of the target more difficult. Thus, the reduction of spatial uncertainty appears to enhance detection.

It has been proposed that illusory contours that are induced by edges and those induced by the ends of lines may be mediated by similar mechanisms (see Lesher and Mingolla 1993; Lesher 1995, for reviews). For example, the effect of contrast polarity (Prazdny 1983; Hershberger and Stallard 1984), the effect of spatial factors on the strength of the illusion (Lesher and Mingolla 1993; Salvano-Pardieu 2000) and their neural mechanisms (Peterhans et al 1986; Peterhans and von der Heydt 1989; von der Heydt and Peterhans 1989; Heitger et al 1992; Peterhans and Heitger 2001) seem to be similar. However, this assumption has not been explicitly tested, and the question whether the two kinds of illusory contour (those induced by edges and those induced by lines) are two variants of the same illusory phenomenon or two different phenomena remains. One conclusion that might be drawn from the results of the two experiments reported above is that the absence of subthreshold summation with an Ehrenstein figure reflects the existence of different mechanisms which mediate the illusory contours in the Ehrenstein figure (end-line induced) and the Kanizsa figure (edge induced). Although various models suggest that contour integration should be effective even if the inducers are not collinear, as in the Ehrenstein figure (Grossberg and Mingolla 1985a, 1985b; Peterhans and von der Heydt 1989; Grossberg 1994; Gove et al 1995), a key difference between the two figures remains the presence or absence of collinearity between the inducing elements and the illusory contour.

In the Kanizsa figure, with which subthreshold summation had previously been reported, the illusory contours are collinear with the edges of the pacman-shaped inducers. Therefore, a target that superimposes the illusory contour will also be collinear with the edges of the inducing elements. In contrast, with the Ehrenstein figure, the illusory contour (and hence a target that superimposes it) is not collinear with the inducing arms of the figure. However, neurophysiological studies of V1 neurons and human psychophysical evidence (Kapadia et al 2000) suggest that detection should also be facilitated inside the illusory area of the Ehrenstein figure and not just around it. Therefore, in this third experiment the target was located across the illusory surface of the Ehrenstein figure, rather than on the illusory contour, such that it was collinear with the inducing arms of the figure. This provides a direct analogue to experiments conducted with the Kanizsa square (eg Dresp and Bonnet 1995).

\section{Experiment 3}

\subsection{Method}

4.1.1 Participants. Six participants (four female and two male), aged between 22 and 45 years, took part in this experiment. All were naive as to the purpose of the present study and all but two had already taken part in psychophysical tasks. All had normal or corrected-to-normal acuity. 
4.1.2 Stimuli and experimental design. As in the second experiment, the stimuli were viewed binocularly on a monochrome computer screen and were generated with a VSG 2/3 graphics card. The three stimulus conditions (Ehrenstein, dot, and control) were the same as in experiment 2. In the first condition, an Ehrenstein figure with eight lines of equal length in a collinear two-by-two configuration was presented, which generated a clear diamond-shaped illusory contour. In the second condition, eight dots were presented instead of the lines, producing no illusory contours. In the final control condition, the target was presented alone. In each of the three conditions, participants had to detect a straight line in one of two target location conditions. The target was either superimposed on the illusory contour or it was located on the illusory surface between two collinear inducers (in the Ehrenstein condition, and in the same locations in the other two conditions). Therefore, there were six conditions, obtained by orthogonal crossing of the two factors (condition and target location), as shown in figure 10. Each participant took part in all six conditions, and the six blocks were randomised for each participant.

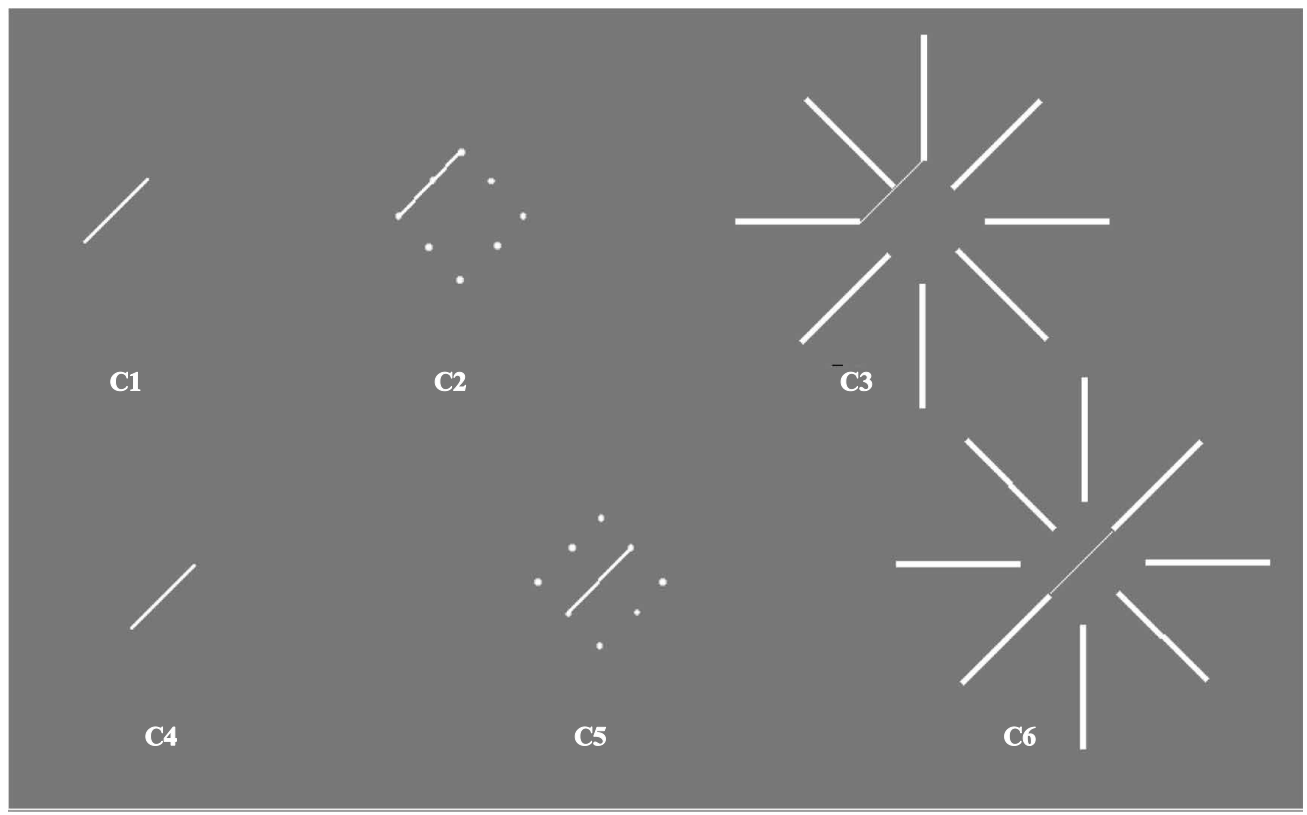

Figure 10. The six stimulus conditions used in experiment 3. Subjects had to detect a target line that was brighter than the background in two different locations: either presented on the contour, or presented on the surface of the figure in the Ehrenstein condition. The target line was either alone in the control condition $(\mathrm{C} 1)$, or joining two dots in an eight-dot condition (C2), or superimposed on the illusory contour and joining the terminations of two inducers in the Ehrenstein condition (C3). When the target line was on the surface of the figure in the Ehrenstein condition, it was either alone in the control condition (C4), or joining two dots across the fixation point (C5), or joining two collinear arms of the Ehrenstein figure (C6). The orientation of the target line was oblique and tilted to the left or right in all conditions.

4.1.3 Procedure. A two-alternative forced-choice (2AFC) staircase procedure was used for threshold determination. There were two target-location conditions. In the contour condition, the straight oblique target line was located on the illusory contour of the Ehrenstein figure (or in the same location in the dot and control conditions) and participants were required to indicate whether the target appeared to the upper-left or upper-right of the fixation point. In the collinear condition, the straight oblique target line was presented across the illusory surface and collinear with two arms of the Ehrenstein figure (or in the same location in the dot and control conditions). 
In this condition, the target passed through the location of the fixation point, and participants were required to indicate whether the top of the target was to the left or the right of the fixation point (see figure 10). The choice of a 2AFC procedure allowed comparison of the same two oblique target lines in the two different location conditions. Participants were required to give their response via the left or right keys of a response box (Cambridge Research Systems CT3). The characteristics of the staircase procedure (other than being $2 \mathrm{AFC}$ as opposed to 4AFC) were the same as in experiment 2. All characteristics of the stimuli, other than those described above, were identical to those in experiment 2. As in the previous experiments, each condition was presented 4 times. The entire experiment lasted approximately $1 \mathrm{~h}$. All other characteristics of this experiment remained the same as in experiment 2 .

\subsection{Results}

The results were analysed with a three-way repeated-measures ANOVA: stimulus condition (Ehrenstein, dot, or control), target location (contour or collinear), and session (1 to 4).

4.2.1 Main effect of stimulus condition. As shown in figure 11, the threshold for target detection was lower in the dot condition $\left(40.82 \mathrm{~cd} \mathrm{~m}^{-2}\right)$, than in the Ehrenstein condition $\left(40.98 \mathrm{~cd} \mathrm{~m}^{-2}\right)$, and was highest in the control condition $\left(41.47 \mathrm{~cd} \mathrm{~m}^{-2}\right)$. The ANOVA showed a significant main effect of stimulus condition $\left(F_{2,10}=66.36\right.$, $p<0.001)$. Planned comparisons showed that the detection threshold was significantly lower in the dot condition than in the Ehrenstein condition $\left(F_{1,5}=8.66, p<0.05\right)$. Furthermore, the threshold for the Ehrenstein condition was significantly lower than in the control condition $\left(F_{1,5}=66.07, p<0.001\right)$.

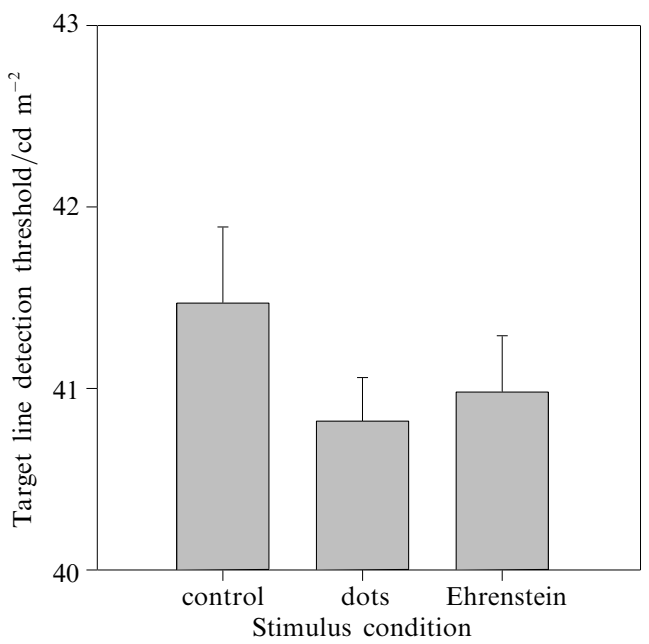

Figure 11. Mean detection thresholds and standard errors for stimulus condition (control, dots, Ehrenstein). Results are averaged across six participants.

4.2.2 Main effect of target location. As shown in figure 12, the threshold for target detection was lower when the target was on the surface of the figure and collinear with the inducers $\left(40.95 \mathrm{~cd} \mathrm{~m}^{-2}\right)$, than when it was on the illusory contour (not collinear with the inducers) $\left(41.24 \mathrm{~cd} \mathrm{~m}^{-2}\right)$. This difference is statistically significant $\left(F_{1,5}=12.47\right.$, $p<0.05)$.

4.2.3 Interaction between stimulus condition and target location. No interaction was found between stimulus condition and target location (see figure 12). Regardless of whether the target is on the illusory contour or collinear with the inducers, detection of the target is not facilitated by the Ehrenstein figure. Performance was no better with the Ehrenstein illusion than with the dot configuration with targets either on the contour or collinear with the inducers. Finally, no main effect or interactions were obtained for session. 


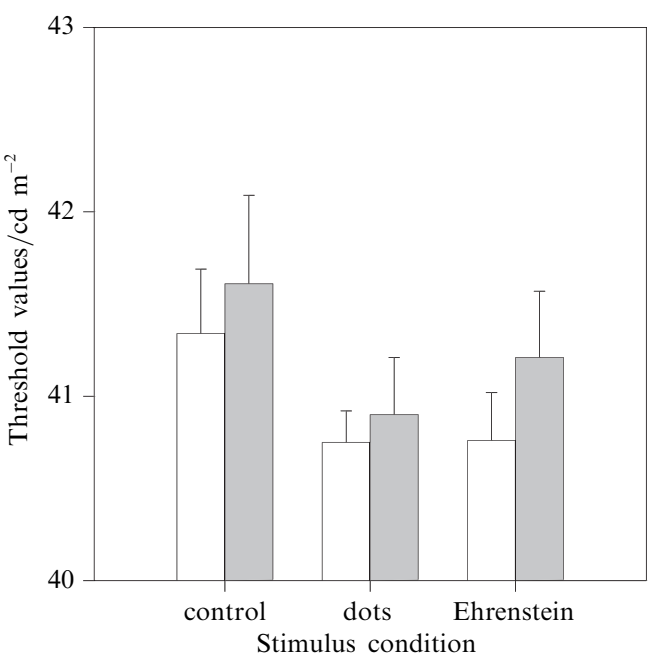

Location of the target surface contour

Figure 12. Mean detection thresholds and standard errors for target line location (on the contour or on the surface) and stimulus condition (control, dots, Ehrenstein). Results are averaged across six participants.

\subsection{Discussion}

In this third experiment, as in the previous two experiments, detection was significantly better in the dot condition than in the Ehrenstein condition. In addition, we found no facilitation of detection, compared to the dot condition, whether the target was superimposed on the illusory contour or presented collinear with the inducing arms and across the illusory surface of the Ehrenstein figure. So, again we found no evidence of subthreshold summation, despite equating the Ehrenstein figure with the Kanizsa figure with respect to the collinearity of the target with the inducing elements.

This may suggest that there are two different mechanisms for the generation of illusory contours, one responsible for line-end-induced contours and the other for edgeinduced contours. However, to confirm the differences suggested here, we need to replicate these experiments using edge-induced illusory contours (such as those produced by the Kanizsa figure) and demonstrate subthreshold summation under similar condition to those investigated here.

\section{General discussion}

On the basis of previous studies it was hypothesised that summation with the illusory contour of an Ehrenstein figure should facilitate detection of a physical target line superimposed on the illusory figure. None of the three experiments reported here showed any evidence of such facilitation. In all three experiments detection of the target line was better when the target was superimposed on the illusory figure, joining two arms of the figure, than when the target was simply presented on a blank screen. In each experiment, performance was also compared to a condition in which the arms of the Ehrenstein figure were replaced by small dots. This stimulus provided accurate spatial cues to the location of the target lines, but generated no illusory contours. This condition was designed to check that any facilitation of detection was not simply a result of a reduction of spatial uncertainty provided by the arms of the Ehrenstein figure. It was therefore expected that subthreshold summation would lead to better detection in the Ehrenstein condition than in the dot condition. However, in contrast, it was found that performance was always better in the dot condition than in the Ehrenstein condition. This clearly seems to demonstrate that it is the provision of spatial cues, rather than the presence of the illusory contour, that is facilitating detection of the target. The reduction of spatial uncertainty allows observers to focus their attention on the positions in which the target may appear. It is known that focusing attention results in an enhanced response and selectivity of cells that monitor 
stimuli appearing in relevant locations (Spitzer et al 1988), as well as a decreased response (inhibitory effect) of cells processing stimuli from non-monitored locations (Moran and Desimone 1985). These changes in neuronal activity, which result from focusing attention, may mediate the enhanced detection that we have observed. This may explain both why the control condition is worst and why the dot condition was found to be better than the Ehrenstein condition, since the dots provide an even more precise cue than the arms of the Ehrenstein figure. These results provide no evidence of subthreshold summation between an illusory contour generated by an Ehrenstein figure and the physical target line.

In the first experiment, the illusory figure was generated by a four-arm Ehrenstein configuration. Such a figure produces a relatively weak and ambiguous illusory contour, and it was thought that this may account for failure to observe subthreshold summation in this experiment. The second experiment was therefore conducted with an eight-arm Ehrenstein figure, which produced a stronger and unambiguous diamondshaped illusory contour. However, the results of this second experiment were the same as of the first, and no evidence of subthreshold summation was found.

The results of the first two experiments appear to contradict the findings of previous studies, which have reported evidence of subthreshold summation between illusory contours and physical target lines. These previous studies have used Kanizsatype figures, in which the illusory contour is generated by edges, rather than by the ends of lines as in the Ehrenstein figures studied here. Although various models suggest that collinearity of inducers should be unimportant (Grossberg and Mingolla 1985a, 1985b; Peterhans and von der Heydt 1989; Grossberg 1994; Gove et al 1995), this remains an important difference between the two types of illusion. In the case of a Kanizsa figure, the illusory contour, and hence the target, is collinear with the inducing elements. In the case of the Ehrenstein figure, the illusory contour is not collinear with the inducing arms. Therefore, a target superimposed on the illusory contour, as in the first two experiments, would not be collinear with the inducing arms either. The third experiment was therefore designed to equate the Ehrenstein figure with the Kanizsa figure with respect to collinearity between the inducing elements and the target line. This placed the target on the illusory surface of the Ehrenstein figure. The results of this third experiment still showed that detection was facilitated more by the dots than by the Ehrenstein figure.

There appears to be a contradiction between the present findings with the Ehrenstein illusion and previous reports for Kanizsa-type figures. One explanation of this might be that the underlying mechanisms that lead to the generation of the illusory contours in these two types of illusion are not in fact the same, and the illusory contours have different characteristics. Consequently, the mechanism which generates the illusory contours in Kanizsa-type figures does give rise to subthreshold summation with a physical target, while the mechanism that generates the illusory contours in the Ehrensteintype figure does not. This explanation would, however, be at odds with the various models in which it has been postulated that the illusory contours in both types of figure are generated by the same mechanisms. For this reason, this explanation warrants further investigation. This might include a much more direct comparison between the conditions under which subthreshold summation has been reported with the Kanizsa figure, and the conditions that have been used in the present study to investigate the Ehrenstein illusion.

\section{Conclusion}

The present results are unexpected in the light of previous research on the Kanizsa square. They demonstrate the need for further research on the conditions under which subthreshold summation can be elicited between illusory contours and physical targets. 
The failure to observe subthreshold summation in this study may reflect differences in the underlying mechanisms responsible for the generation of illusory contours induced by lines (Ehrenstein) and edges (Kanizsa). An alternative possibility may be that the apparent subthreshold summation reported in previous studies is in fact related to reduced spatial uncertainty. This might be investigated by comparing the dot condition used in this study with a Kanizsa figure, which would allow a direct comparison with the original stimulus for which subthreshold summation has been reported.

Acknowledgments. We would like to thank "la region centre" and the University of Tours, which provided funding for conducting these experiments. We wish to thank the anonymous referees for their constructive comments on the original submission, Michel Isingrini and Roger Fontaine for their support, and Walter Ehrenstein for his assistance with the editing of this article.

\section{References}

Coren S, Porac C, Theodor L, 1987 "Set and subjective contour", in The Perception of Illusory Contours Eds S Petry, G E Meyer (New York: Springer) pp 237-245

Deters-Brüggemann H, Meinhardt G, 1994 "A hysteresis model for the temporal dynamics in the Ehrenstein illusion" Perception 23 Supplement, 103-104

Dresp B, Bonnet C, 1995 "Subthreshold summation with illusory contours" Vision Research 35 $1071-1078$

Dresp B, Grossberg S, 1997 "Contour integration across polarities and spatial gap: from local contrast filtering to global grouping" Vision Research 37 913-924

Dresp B, Grossberg S, 1999 "Spatial facilitation by color and luminance edges: boundary, surface, and attentional factors" Vision Research 393431 - 3443

Dresp B, Salvano-Pardieu V, Bonnet C, 1996 "Illusory form with inducers of opposite contrast polarity: Evidence for multi-stage integration” Perception \& Psychophysics $58111-124$

Ehrenstein W, 1941/1987 "Über Abwandlungen der L. Hermannschen Helligkeitserscheinung" Zeitschrift für Psychologie 150 83-91 [translated by A Hogg "Modification of the brightness phenomenon of L. Hermann", in The Perception of Illusory Contours Eds S Petry, G E Meyer (1987, New York: Springer) pp 35-39]

Ejima Y, Takahashi S, 1988 "Illusory contours induced by isoluminant chromatic patterns" Vision Research $281367-1377$

Gove A, Grossberg S, Mingolla E, 1995 "Brightness perception, illusory contours and corticogeniculate feedback" Visual Neuroscience 12 1027-1052

Grossberg S, 1994 "3-D vision and figure - ground separation by visual cortex" Perception \& Psychophysics $\mathbf{5 5} 48-120$

Grossberg S, Mingolla E, 1985a "Neural dynamics of form perception: Boundary completion, illusory figures, and neon color spreading" Psychological Review 92 173-211

Grossberg S, Mingolla E, 1985b "Neural dynamics of perceptual grouping: Textures, boundaries, and emergent segmentations" Perception \& Psychophysics $38141-171$

Hamada J, Nakahashi T, 1995 "Relationship between form and brightness in the Ehrenstein illusion" Perception 24 Supplement, 79

Heider B, Spillmann L, Peterhans E, 2002 "Stereoscopic illusory contours-cortical neuron responses and human perception" Journal of Cognitive Neuroscience 141018 - 1029

Heitger F, Rosenthaler L, Heydt R von der, Peterhans E, Kubler O, 1992 "Stimulation of neural contour mechanisms: From simple to end-stopped cells" Vision Research 32 936-978

Hershberger W, Stallard S, 1984 "Contrast variability lightens subjective figures" Perception \& Psychophysics $3692-94$

Heydt R von der, Peterhans E, 1989 "Mechanisms of contour perception in monkey visual cortex. I: Lines of pattern discontinuity" Journal of Neuroscience 91731 - 1748

Huxlin K R, Saunders R, Marchionini D, Pham H-A, Merigan W H, 2000 "Perceptual deficits after lesions of inferotemporal cortex in macaques" Cerebral Cortex 10 671-683

Kanizsa G, 1955/1987 "Margini quasi-percettivi in campi con stimulazione omogenea" Rivista di Psychologia 49 7-30 [translated into English as "Quasi-perceptual margins in homogeneously stimulated field", in The Perception of Illusory Contours Eds S Petry, G E Meyer (1987, New York: Springer) pp 40-49]

Kapadia M K, Westheimer G, Gilbert C D, 2000 "Spatial distribution of contextual interactions in primary visual cortex and in visual perception" Journal of Neurophysiology $842048-2062$

Kellman P, Loukides M, 1987 "An object perception approach to static and kinetic subjective contours", in The Perception of Illusory Contours Eds S Petry, G E Meyer (New York: Springer) pp $151-164$ 
Kennedy J M, 1976 "Sun figure: an illusory diffuse contour resulting from an arrangement of dots" Perception 5 479-481

Kulikowski J J, King-Smith P E, 1973 "Spatial arrangement of line, edge, and grating detectors revealed by subthreshold summation" Vision Research $131455-1478$

Lesher G, 1995 "Illusory contours: Toward a neurally based perceptual theory" Psychonomic Bulletin \& Review $131-50$

Lesher G, Mingolla E, 1993 "The role of edges and line-ends in illusory contour formation" Vision Research $332253-2270$

Lieberman H R, Pentland A P, 1982 "Microcomputer-based estimation of psychophysical thresholds: The best PEST" Behaviour Research Methods and Instrumentation 14 21-25

Meese T S, 1995 "Using the standard staircase to measure the point of subjective equality: a guide based on computer simulation" Perception \& Psychophysics 57 267-281

Moran J, Desimone R, 1985 "Selective attention gates visual processing in the extra-striate cortex" Science $229782-784$

Pentland A, 1980 "Maximum likelihood estimation: The best PEST" Perception \& Psychophysics $28377-379$

Peterhans E, Heitger F, 2001 "Simulation of neuronal responses defining depth order and contrast polarity at illusory contours in monkey area V2" Journal of Computational Neuroscience 10 $195-211$

Peterhans E, Heydt R von der, Baumgartner G, 1986 "Neuronal responses to illusory contour stimuli reveal stages of visual cortical processing", in Visual Neuroscience Eds J D Pettigrew, K Sanderson, W Levick (Cambridge: Cambridge University Press) pp 343 - 351

Peterhans E, Heydt R von der, 1989 "Mechanisms of contour perception in monkey visual cortex. II: contours bridging gaps" Journal of Neuroscience $91749-1763$

Poom L, 2001 "Visual summation of luminance lines and illusory contours induced by pictorial, motion, and disparity cues" Vision Research 413805 - 3816

Prazdny K, 1983 "Illusory contours are not caused by simultaneous brightness contrast" Perception \& Psychophysics $34403-404$

Redies C, Spillmann L, Kunz K, 1984 "Colored neon flanks and line gap enhancement" Vision Research $241301-1309$

Salvano-Pardieu V, 2000 "Spatial factors of brightness illusion in the Ehrenstein figure" Perception $29709-720$

Shapley R, Gordon J, 1987 "The existence of interpolated illusory contours depends on contrast and spatial separation", in The Perception of Illusory Contours Eds S Petry, G E Meyer (New York: Springer) pp 109-115

Spillmann L, 1975 "Perceptual modification of the Ehrenstein illusion", in Gestalttheorie in der modernen Psychologie Eds S Ertel, L Kemmler, M Stadler (Darmstadt; Steinkopff) pp 210 - 218

Spillmann L, Dresp B, 1995 "Phenomena of illusory form: can we bridge the gap between levels of explanation?" Perception $241333-1364$

Spillmann L, Fuld K, Neumeyer C, 1984 "Brightness matching, brightness cancellation, and increment threshold in the Ehrenstein illusion" Perception 13 513-520

Spitzer H, Desimone R, Moran J, 1988 "Increased attention enhances both behavioral and neuronal performance" Science $\mathbf{2 4 0} 338$ - 340 


\section{PERCEPTION}

VOLUME 352006

www.perceptionweb.com

Conditions of use. This article may be downloaded from the Perception website for personal research by members of subscribing organisations. Authors are entitled to distribute their own article (in printed form or by e-mail) to up to 50 people. This PDF may not be placed on any website (or other online distribution system) without permission of the publisher. 